\title{
Design and Development of Digital Umlaut and Speed Change Learning System of Intelligent Foreign Language
}

\author{
Yanmei Meng, Weili Chu \\ Qingdao Huanghai University
}

Keywords: Intelligent system, Network protocols, Multimedia processing technology

\begin{abstract}
The intelligent foreign language digital learning system is a set of specialized foreign language teaching equipment and software. It has the characteristics and advantages of digital signal transmission and storage, network sharing of resources, as well as user-friendly operation, etc. This thesis analyzes and studies the whole scheme of digital language learning system, with the design of communication protocol, the design of hardware and the development of software and so on. Through tests, it is shown that the system can be applied to foreign language teaching, which also can explore new ways for the application of computer technology and education as well as teaching.
\end{abstract}

\section{Introduction}

Digital foreign language learning system is the inevitable result of the development of computer network technology and multimedia technology, which is a trend of technology development. The language labs that we used to contact were analog language systems, such as analog phones, cassette recorders and so on. For a long time, our life has been using analog products, but the functions of traditional analog devices have been unable to meet the needs of society. Taking the language lab as an example, the traditional analog language lab has poor sound quality, poor stability, poor teaching interaction with the single function of system. At present[1], the main software and hardware digital language learning system in the market is based on embedded system, which is relatively low cost and practical. Today more mature systems are almost all foreign products, there are only a few domestic companies had research and development in this field. In the new model of language teaching, it will also transform the teaching skills and knowledge model from the teacher-oriented to student-oriented, which will put more focus on changing the training model to develop students' autonomous learning ability and language application ability[2]. In order to change this pattern, it will not be limited in the language lab, it is necessary to construct the campus network based open digital language learning platform, so as to promote the reform of foreign language teaching to improve students' application ability and learning ability as the goal.

The main design goal of this paper is to set up an embedded digital voice teaching system which integrates software and hardware modules, as well as network technology and audio data. 


\section{Related Technical Introduction and System Design}

Overview of Streaming Media Technology

In recent years, streaming media has been widely used in many countries, especially the introduction of the first Internet streaming media player in 1995. The application of Internet streaming media has increased rapidly. The development of Internet communication technology has laid the foundation for the development of streaming media technology. Commercial websites use more streaming media technologies to implement VOD, live music and news, while organizations and enterprises use streaming media technology to realize company introduction, information release and employee training and so on. These applications greatly improve the efficiency of the work, which also can save costs and expenses[3]. With the development of new technologies, such as digital multimedia, network communication and computer software, streaming media player have developed rapidly.

\section{Network Transmission Protocol}

In this system, teachers PC server used Windows operating system, basically it had integrated most of the network protocols; but based on the student terminal embedded processor, considering the limited resource itself, it is necessary for network protocol to have selection and cut, if it is necessary, it also needs to design their own network protocol.

\section{Student Terminal TCP/IP Protocol Selection}

The main function of network communication interface is to provide the service of sending and receiving data packets for the upper layer protocol, which can have many ways to access to the network. In the system of this paper, the method that student terminal is connected to the Internet network is to use RTL8019AS communication interface chip port 11.

The main function of network layer is to encapsulate and process packets. Because the Ethernet protocol is realized at the bottom, and the MAC address of network is used to identify the data transmission, therefore, the system is required to implement the conversion between IP and MAC addresses, namely, to realize address resolution protocol. In TCP/IP group, IP protocol is its core protocol. Only by implementing IP protocol can data be transmitted in different networks. In the TCP/IP family, TCP is a connection-oriented protocol. In order to guarantee the reliability of data transmission, the overspending of network is very large, which may cause the data transmission to have a great delay, as for the requirement of having real-time audio transmission in the system, this kind of delay in most cases will be beyond the tolerance of audio transmission. However, compared with TCP protocol, UDP removes the tedious synchronization, confirmation step and other steps, which can save network spending and it also can improve the data transmission rate. Therefore, in the design of this system, the audio data transmission can be carried out by using UDP protocol.

\section{Overall Framework Design of System}

The digital language learning system must meet the requirements of network, which also should realize the encoding and decoding of multimedia document streams. Therefore, the open digital media structure is adopted in this paper. At the same time, the high performance media architecture integrates many new applications, such as data content, voice, video, etc., which also can construct the digital media data providing methods. The main functions that 
the system needs to implement are real-time transmission performance, flexibility of software programming and higher channel density to meet the requirements of simultaneous data stream as well as voice transmission.

\section{Audio Streaming Protocol}

Because this system used only a small LAN with about more than 40 terminals, thus the quality of network transmission is no longer existed. According to the system's real-time transmission of audio documents, we use real-time transmission protocol RTP in our system. Real time transport protocol can provide a guarantee for real-time data transmission.

Since the packet transmission of teachers and student terminal services exist audio data only, while audio data is from teacher's machine, so CC and CSRC in RTP protocol will have a relatively large redundancy. Due to the data transmission source of digital voice teaching system has special characteristics, we have to improve the transmission efficiency of audio resources, from the framework of RTP protocol, the field can be redesigned, the original protocol is simplified and has the corresponding reduction, thereby it can greatly reduce the redundancy, so as to realize the requirements of the system such as special, streamline and efficient and so on; this paper designed the real-time transmission protocol of audio data that can adapt to the system, which can be called as MyProtocol.

\section{Overall Design of System}

The digital language learning system must meet the requirements of network so as to realize the encoding and decoding of multimedia document streams. Therefore, the open digital media structure is adopted in this paper. At the same time, the high performance media architecture integrates many new applications, such as data content, voice, video, etc., which also can construct the digital media data providing methods. The main functions that the system needs to implement are real-time transmission performance, flexibility of software programming and higher channel density, so as to meet the requirements of simultaneous data stream and voice transmission. The architecture of this system can be shown in Fig. 1.

According to the diagram above, the functions of each part of the system are as follows:

(1) Audio Input. It can support many types of external audio streams, including microphones, tape recorders, etc..

(2) Video Input. It can support many types of external video streams, including camcorders, VCD/DVD, cable TV, etc..

(3) Student terminal. It can realize the function of interaction with students, decoding the data information received from the network or encoding the data on the machine, then sending it to the server through the network, so as to realize the local storage of USB disk.

(4) Host controller / network switch. It can provide the necessary power and network data package for the student terminal.

(5) Teacher computer server. It is the core of the system, which is responsible for converting the external input, audio stream and video stream, as well as the data stored locally by the server into data packets, so as to sent to each student terminal through the network channel. 


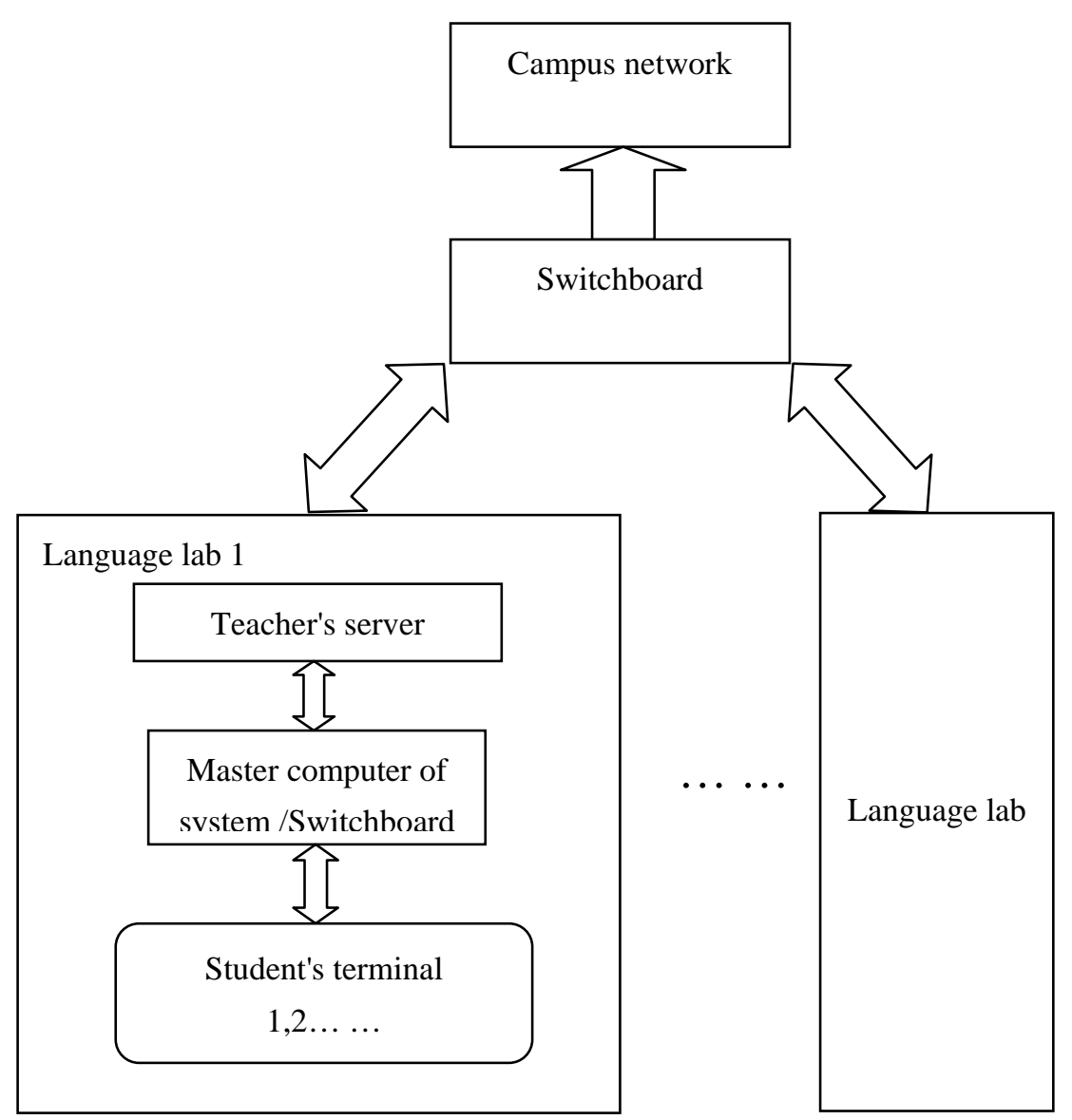

Fig.1 System Framework Digram of Digital Umlaut and Speed Change Learning System of Intelligent Foreign Language

\section{Conclusion and Prospect}

In China, digital language learning system is still in the developing period. As for the current technology and digital products on the market, it can basically meet the needs of users, features of products are more complete, more compatible. The existing IT technology can effectively integrate text, with video, audio, images and other teaching resources together, however, manufacturers have different ways and means of technical implementation[4]. Therefore, manufacturers have different understanding on design concepts of the digital language learning system, how to apply the technology to the integration of foreign language curriculum teaching, so as to make it more in line with the digital language learning environment for foreign language learning and teaching rules is the key issue to consider various manufacturers. Based on the above understanding, the author thinks that it is imperative to design and develop high-level digital language learning system.

The fundamental purpose of digital language learning system to achieve digital storage and transmission of language teaching, so as to realize cyber source sharing; therefore, the system should be established on the basis of the current mainstream network, which is the basic requirement, moreover, it also should comply with the requirements of data transmission according to standard TCP/IP protocol, the student terminals can should have independent IP address. Only in this way, the student terminals can have access to the campus network source directly. Under such environment, we have studied and designed digital language learning system. In this paper, it presents the overall structure of the system, according to the 
characteristic of the system to design and implement MyProtocol of the system. It also can solve the problems that the original audio equipment may occur, such as "delay or breaking" under the Ethernet environment in order to get clear voice, real-time and smooth transmission. The designed student terminal hardware platform implements the transplant of uC-OS-II of the embedded system kernel, which writes and develops network protocol drivers as well as the related applications.

\section{References}

[1] Advances in Chinese Document and Text Processing[M]. World Scientific, 2017.

[2] Zhang X, Li H, Zhao J, et al. Emergency Communication Network Design and Key Technology Research Based on Ad Hoc Network[J]. Journal of Applied Science and Engineering Innovation, 2016, 3(6): 213-216.

[3] Naboulsi M A. Hands on steering wheel vehicle safety control system: U.S. Patent 9,526,447[P]. 2016-12-27.

[4] Xie D, Zhang Y, Xiao J, et al. Training Graduate Students of the Computer Science and Technology Field in China[J]. Journal of Applied Science and Engineering Innovation, 2016, 3(2): 49-51. 